OPEN ACCESS

Edited by:

Fang-Bao Tian,

University of New South Wales

Canberra, Australia

Reviewed by:

Qiang Liu,

China University of Petroleum, China

Hadi Rostamzadeh,

Sharif University of Technology, Iran

${ }^{*}$ Correspondence:

Wei Gao

weigao@seu.edu.cn

Specialty section:

This article was submitted to

Process and Energy Systems

Engineering,

a section of the journal

Frontiers in Energy Research

Received: 28 April 2021

Accepted: 31 May 2021

Published: 17 June 2021

Citation:

Li L, Tao J and Gao W (2021)

Experimental Study on the Dynamic Heat Transfer Characteristics of a

Mechanically Pumped Two-phase

Cooling Loop.

Front. Energy Res. 9:701805.

doi: 10.3389/fenrg.2021.701805

\section{Experimental Study on the Dynamic Heat Transfer Characteristics of a Mechanically Pumped Two-phase Cooling Loop}

\author{
Lei Li, Jianyun Tao and Wei Gao* \\ Key Laboratory of Energy Thermal Conversion and Control of Ministry of Education, School of Energy and Environment, \\ Southeast University, Nanjing, China
}

The present research designs a mechanically pumped cooling loop system and conducts an experimental investigation on the dynamic heat transfer characteristics of the system. The effects of the start-up heat load and heat load variation on the dynamic characteristics of the system are analyzed. The results indicate that during the start-up period, as the heat load rises, the temperature overshoot and duration time of adjustment decrease, while the pressure oscillation amplitude increases. During the regular operation period, as the heat load increases, the temperature at the evaporator outlet gradually increases until it approaches the saturation temperature and then remains constant. The pressure evolution at the evaporator outlet can be divided into four stages: steady state, drastic oscillation, damped oscillation, and slight oscillation.

\footnotetext{
Keywords: two-phase cooling, heat transfer characteristics, temperature overshoot, adjustment time, pressure oscillation
}

\section{INTRODUCTION}

The development of very large-scale integration (VLSI) contributes to the extensive attention given to thermal control technologies for equipment with a high power density, including data center servers, avionic devices, hybrid vehicles, microwave military systems, and high-energy laser diodes (Thome, 2006). Since the power density of these devices approaches $300 \mathrm{~W} / \mathrm{cm}^{2}$, the research emphasis has shifted from traditional air cooling and liquid cooling to two-phase cooling with a higher heat dissipation potential (Lee et al., 2014). Because of the considerable potential of two-phase cooling technology, space agencies of various countries have devoted their efforts to studying twophase thermal control systems (Chen et al., 2010; Liu et al., 2013) for the future requirements of spacecraft and space-borne equipment with a high power density. Particularly, the mechanically pumped cooling loop (MPCL) system has been a research hotspot in recent years in the active thermal control of space applications due to its better thermal performance and higher stability (Meng et al., 2020).

Nowadays, several efforts devoted to investigate the vapor-liquid and liquid-liquid two-phase flow by using numerical method (Zhou et al., 2019) and experimental test (Chen et al., 2013). By these available experimental and numerical data, it is well known that two-phase flow heat exchange systems tend to cause temperature and pressure pulses when converting from a single phase to two phases. If the pulse is too large, the system becomes unstable and can even cause fatal damage (Jie et al., 2008). Although there is no problem associated with start-up obstacles in the MPCL system, there are few studies on the start-up characteristics of the system change from single-phase heat 
TABLE 1 | Summary of two-phase cooling loop research.

\begin{tabular}{|c|c|c|c|}
\hline $\begin{array}{l}\text { Two-phase } \\
\text { cooling loop }\end{array}$ & Advantage & Disadvantage & Main finding \\
\hline Loop heat pipe & $\begin{array}{l}\text { Large heat capacity and heat-transfer } \\
\text { distance Shi et al. (2021) }\end{array}$ & $\begin{array}{l}\text { The payload temperature varies with the } \\
\text { payload power Vasiliev (2005) }\end{array}$ & $\begin{array}{l}\text { The high capillary pressure generated by the wick can be } \\
\text { employed to extend the horizontal heat transfer distance as well } \\
\text { as overcome gravity. Maydanik (2005) }\end{array}$ \\
\hline Loop thermosyphon & $\begin{array}{l}\text { Low power consumption Wang et al. } \\
\text { (2018) }\end{array}$ & $\begin{array}{l}\text { Low heat removal capacity Zhu and Yu } \\
\text { (2019) }\end{array}$ & $\begin{array}{l}\text { When operating in the vertical orientation, higher R134a fill ratios } \\
\text { are more effective. Kloczko and Faghri (2020) }\end{array}$ \\
\hline MPCL & $\begin{array}{l}\text { High heat removal capacity; Compact } \\
\text { Meng et al. (2020) }\end{array}$ & High power consumption Ma et al. (2016) & $\begin{array}{l}\text { A lower evaporative temperature can cause higher temperature } \\
\text { overshoot. Jie et al. (2008) }\end{array}$ \\
\hline
\end{tabular}

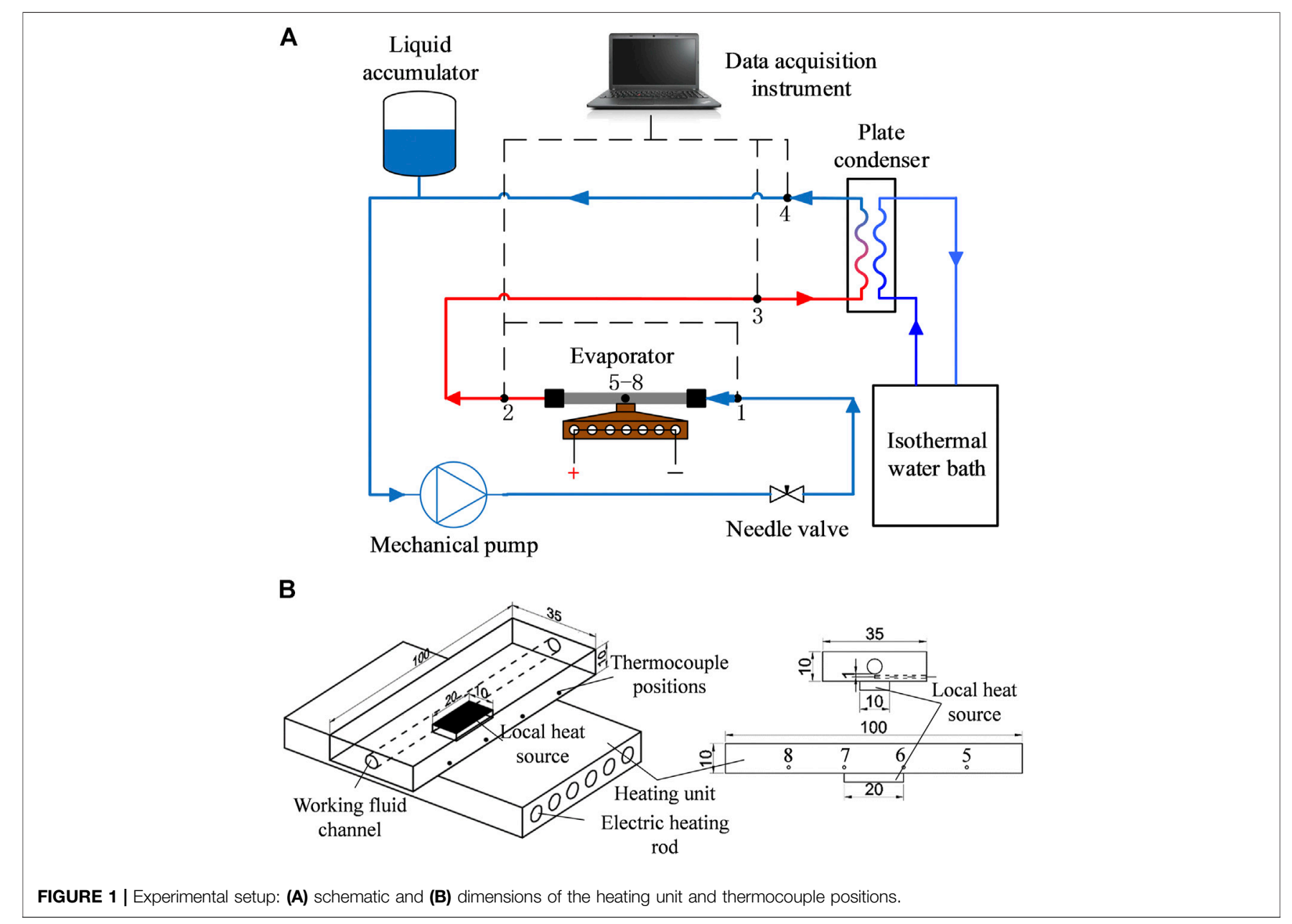

transfer to two-phase heat transfer. Therefore, further research is needed to supplement the factors affecting pulse size. Shi et al. (2021) conducted a numerical simulation of a loop rotating heat pipe, exploring the effects of heating power and rotation speed on flow behavior and heat transfer characteristics. Zhang et al. (2021) conducted an experimental study on temperature control of a mechanically pumped two-phase cooling loop for the active cooling of high-heat-flux microelectronic devices via microchannel boiling, in which the thermal control system is configured with the active disturbance rejection control. Meng et al. (2020) reported an experimental study on the transient behaviors of the mechanically pumped two-phase loop with a novel accumulator, focusing on the temperature variation during start-up and heat sources load-on/off. A brief comparison of the two-phase cooling loop systems is summarized in this section, as shown in Table 1.

In general, the current research on pumped two-phase heat exchange systems mainly focuses on testing the performance of the system and discussing the temperature control system. However, there are few studies on the heat transfer 
mechanism at different stages of the system, and many factors affect the cycle performance of the system, which limits the availability of research data in this area. Besides, the pressure oscillation during temperature overshoot under the start-up process is rarely reported, while the mechanism research of pressure oscillation is of great significance in strat-up planning of two-phase loop.

In this paper, an MPCL system is designed to further study the heat transfer characteristics of two-phase cooling systems. To describe the thermal performance of the MPCL system, an experiment to measure the system temperature and pressure is conducted. The control variable method (CVM) is utilized to study the effects of the heat load on the dynamic heat transfer characteristics of the system. Furthermore, the start-up characteristics and thermal response characteristics to variations in the heat load are analyzed.

\section{DESCRIPTION OF THE EXPERIMENT}

\section{Experimental Device}

To investigate the dynamic heat transfer characteristics of an active two-phase cooling system, the MPCL system shown in Figure 1 is designed in this paper. The experimental device consists of a two-phase cooling loop, power supply system, and data acquisition system. The two-phase cooling loop is a carrier for transferring the heat of the working medium. The power supply system provides a heat source with a high power density to simulate heat generation by an electronic chip. Moreover, the measurement and recording of temperature and pressure are carried out through the data acquisition system.

The two-phase cooling loop is composed of a mechanical pump, an isothermal water bath, a liquid accumulator, a needle valve, a plate condenser, and an evaporator. The vacuumization and the charge processes of the two-phase cooling loop are completed by a rotary-vane vacuum pump (2XZ-4) and an auxiliary charging device, respectively. The working medium utilized here is acetone. A liquid accumulator with a capacity of $0.5 \mathrm{~L}$ is connected to the system not only to maintain the pressure stability of the system by recycling the expanding medium but also to adjust the pressure of the system through the temperature control unit. Therefore, the charging capacity is set to $0.45 \mathrm{~L}$ to fill the two-phase cooling loop and leave a certain amount of margin. The two-phase flow loop is powered by a mechanical pump $(Q=0 \sim 2 \mathrm{~L} / \mathrm{min}, P=0 \sim 4$ bar $)$. The evaporator is an aluminum heat sink with a size of $100 \mathrm{~mm} \times 35 \mathrm{~mm}$ $\times 10 \mathrm{~mm}$, and it contains a channel with a diameter of $5 \mathrm{~mm}$ to provide access to the working medium. The cooling capacity of the plate condenser is supported by an isothermal water bath, and the evaporation temperature of the system is controlled by the temperature control unit for the liquid accumulator. In the experiment, the evaporation temperature ranges from $37^{\circ} \mathrm{C}$ to $57^{\circ} \mathrm{C}$ with a step of $10^{\circ} \mathrm{C}$, and the fluid temperature at the exit of the condenser is set to 2,11 , and $20^{\circ} \mathrm{C}$.

The power supply system includes an adjustable AC voltage regulator, a power meter, several electrical heating rods, and two aluminum blocks. A larger block with a size of $100 \mathrm{~mm} \times 35 \mathrm{~mm}$ $\times 10 \mathrm{~mm}$ is the heating unit (shown in Figure 1), in which six holes with a diameter of $6 \mathrm{~mm}$ are fabricated to install electrical heating rods. The smaller block with a size of $10 \mathrm{~mm} \times 20 \mathrm{~mm}$ is the local heat source that simulates the chip. To reduce the contact thermal resistance, liquid metal heat conductors encapsulated by thermally conductive adhesive are arranged between the heating unit and the heat sink base. The power input of the heating unit can be controlled by adjusting the AC voltage regulator and is displayed in real time on the power meter. In the experiment, the heat load is augmented in steps of $75 \mathrm{~W}$ until the heat transfer limitation is reached to study the dynamic response characteristics of the system under different operating conditions.

The data acquisition system, which aims to monitor and record the pressure, temperature, and flow rate, includes several thermocouples (K-type, accuracy $\pm 1^{\circ} \mathrm{C}$ ), a data acquisition instrument (Agilent 34970A), a PC, two Setra pressure sensors (accuracy 0.1\%), and an ultrasonic flowmeter. Several thermocouples are arranged at the entrance and exit of each main component. To understand the dynamic heat transfer process of the evaporator, four thermocouples are evenly located $1 \mathrm{~mm}$ below the center channel with a pitch of $20 \mathrm{~mm}$ (see Figure 1B). The flow rate of the system is displayed on an ultrasonic flow meter in real time. The Setra pressure sensors are placed at the inlet and outlet of the evaporator to study the dynamic thermal response of the system. All of these signals are acquired by an Agilent acquisition instrument and then recorded on a PC.

\section{Data Reduction}

The effective convective heat transfer coefficient is used to characterize the heat transfer performance of the evaporator, which is defined as

$$
h=\frac{q_{w}}{A_{w}\left(T_{w}-T_{s}\right)}
$$

where $q_{\mathrm{w}}$ is the effective heat transfer amount of the working medium, $T_{\mathrm{w}}$ is the inner-wall temperature of the channel equal to the average temperature of the thermocouples located at the evaporator, $A_{\mathrm{w}}$ is the effective heat-transfer area of the channel, and $T_{s}$ is the average temperature of the working medium. Considering the variable heat flux in the horizontal direction of the evaporator due to the limitation of the thermal conductivity, the average temperature of the working medium can be calculated as

$$
T_{s}=\frac{T_{\text {in }}+T_{\text {out }}}{2}
$$

where the subscripts represent the temperature of the working medium in the inlet and outlet of the system.It is noteworthy that heat loss is inevitable in a practical experiment, and the effective heat transfer amount of the working fluid is given by

$$
q_{w}=q_{n e t} \cdot \eta
$$

where $q_{\text {net }}$ is the total heat supplied by the power system and $\eta$ is the heating efficiency accounting for the heat loss to the ambient 

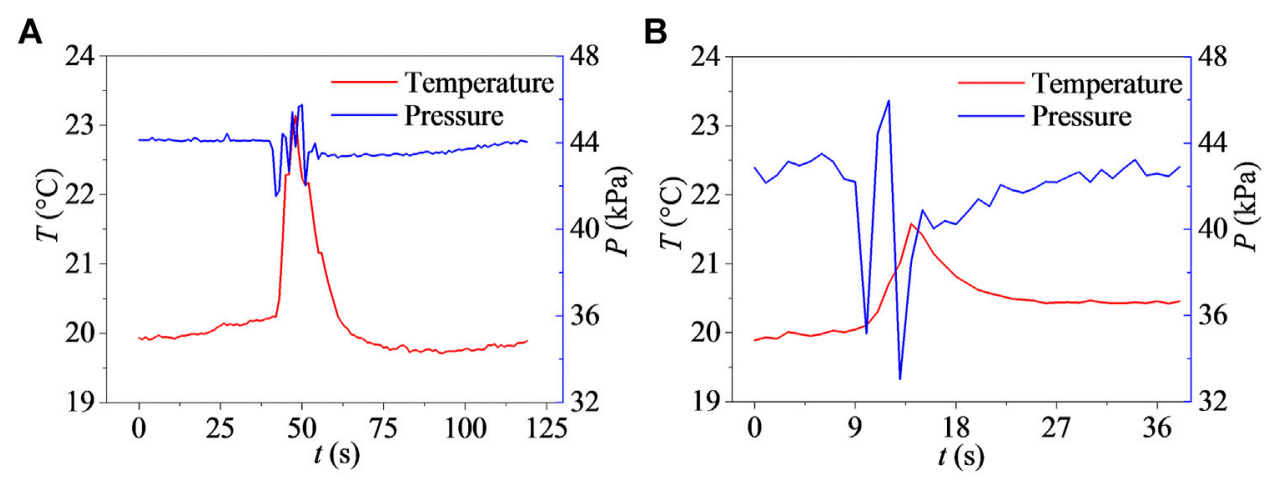

FIGURE 2 | Effect of the initial heat load on the dynamic start-up characteristics: (A) 50 W and (B) 100 W.

environment, which is set to $95 \%$ hereafter comparing the heat dissipation of the fluid with the amount of heating many times.

\section{RESULTS AND DISCUSSIONS}

The study of the heat transfer characteristics of the MPCL system focuses on investigating the start-up characteristics and variablecondition characteristics. The exploration of start-up characteristics aims to predict potential problems and develop corresponding preventive and improvement measures. The investigation of the dynamic thermal response to the variable thermal load is intended to better understand the influencing mechanism of the thermal load on the performance of the MPCL system, which provides a reference for the optimal operation of the system.

\section{Start-Up Characteristics}

Studies on the start-up characteristics of the system focus on the pulse conditions caused by the transition of the system from a single-phase state to a two-phase state, including the amount of temperature overshoot, duration time before stabilization, and amplitude of pressure oscillations. The temperature overshoot can be defined as the value of the instantaneous temperature rise at the evaporator outlet when the system transitions from a single phase to two phases. The duration time can be defined as the time from the start of an overshoot to temperature balance, and the amplitude of the pressure oscillation can be defined as the difference between the amplitudes of adjacent peaks and valleys.

Figure 2 depicts the dynamic variations in the temperature and pressure at the evaporator outlet under various heat loads during the start-up stage of the system. In this process, the mass flow rate of the working medium is $110 \mathrm{~kg} / \mathrm{m}^{2} \mathrm{~s}$, the evaporation temperature is set to $37^{\circ} \mathrm{C}$, the condenser outlet temperature is set to $11^{\circ} \mathrm{C}$, and the initial heating power is set to 50 and $100 \mathrm{~W}$. As shown in the figure, there is a temperature overshoot and a corresponding pressure oscillation at the evaporator outlet during the start-up period. The occurrence of temperature overshoot is related to the heating power, we have pointed out in our previous study (Deng et al., 2021) that the temperature overshoot reflects that the working fluid is developed from the single-phase to the two-phase state. The temperature overshoot as depicted in Figure $\mathbf{2}$ is identical to the typical transformation of the working fluid state. When the heating module is started, the heat source continuously generates heat, and the temperature of the working medium gradually rises due to heat conduction. Once the superheat of the wall reaches a certain level, the nucleation sites on the channel surface are activated. Before reaching the critical radius of the bubble detachment, it needs to absorb enough heat for bubble nucleation, contributing to the sharp increase in the temperature of the working medium (Wang et al., 2018). After that, the nucleation point will continuously generate new bubbles, and a large amount of latent heat is simultaneously absorbed due to the generation of bubbles, which finally leads to a decrease in temperature. Moreover, acetone has a relatively large gas-liquid density, and the working fluid expands rapidly during the vaporization process in the evaporation section, thereby generating a pressure pulse. In general, bubble generation and motion not only enhance the heat transfer but also lead to pressure oscillation of the system (Liu et al., 2016). It is worth noting that the increase in the heat load leads to a decrease in the temperature overshoot and duration time of the adjustment while resulting in an augmentation in the amplitude of the pressure oscillation. In this work, as the input heat power increases from 50 to $100 \mathrm{~W}$, the temperature overshoot is decreased by $50 \%$, from 3 to $1.5^{\circ} \mathrm{C}$, and the duration time of the adjustment is reduced from 24 to $10 \mathrm{~s}$, while the pressure oscillation amplitude is increased from 4 to $12.5 \mathrm{kPa}$. The explanation is that the gasification rate of the working medium is accelerated with increasing heat load, and more latent heat is absorbed, thus reducing the temperature overshoot. However, because of the acceleration of the gasification process, the gas generated per unit time increases, which contributes to the intensity of pressure oscillations.

\section{Variable-Condition Characteristics}

Research on the variable-condition characteristics of the system is conducted mainly to explore the adaptability to heat load variation during operation. The dynamic variations in the temperature and pressure with a varying heat load are monitored.

To investigate the dynamic thermal response of the MPCL system to the heat load, Figure $\mathbf{3 A}$ shows the dynamic variations 


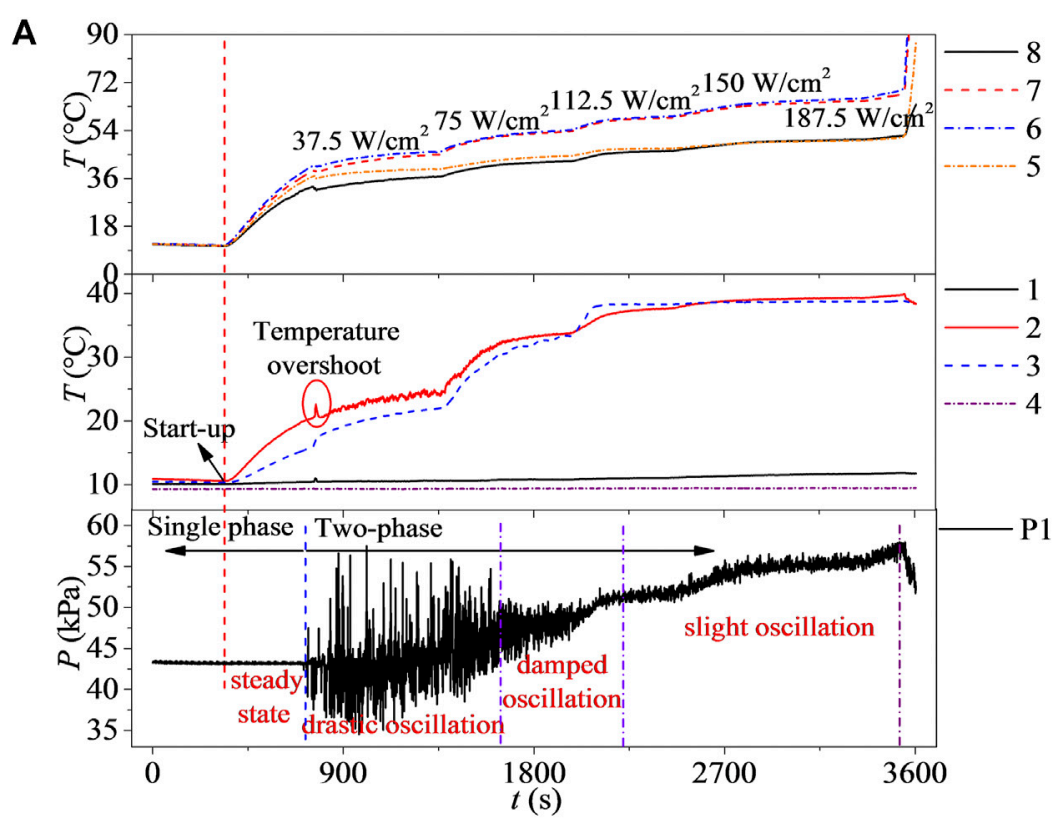

B
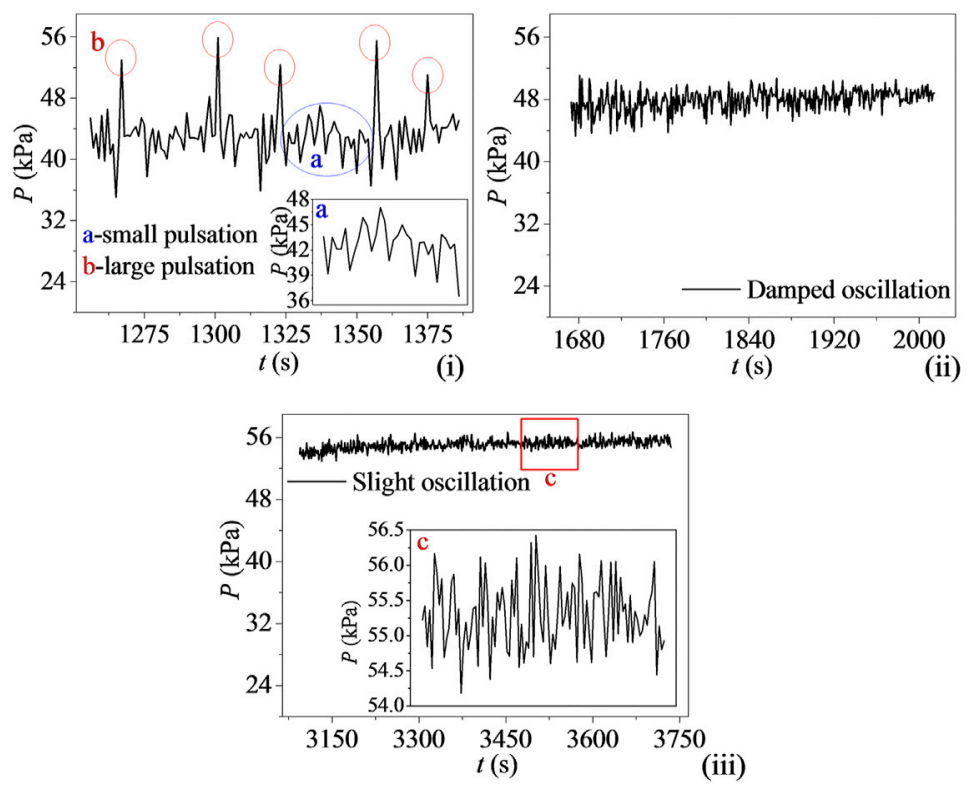

FIGURE 3 | (A) Dynamic thermal response of the MPCL system to a heat load. (B) Pressure fluctuation of the evaporator outlet in two-phase flow: (i) drastic oscillation; (ii) damped oscillation; and (iii) slight oscillation.

in the temperature and pressure at the different positions illustrated in Figure 1. The mass flow rate of the working medium is $110 \mathrm{~kg} / \mathrm{m}^{2} \mathrm{~s}$, and the evaporation temperature and condenser outlet temperature are set to 37 and $11^{\circ} \mathrm{C}$, respectively. The initial heating power of the evaporator is $75 \mathrm{~W}$. When the system reaches a steady state, a heat load of $75 \mathrm{~W}$ is applied to the evaporator until a boiling crisis (i.e., the critical heat flux density) occurs. As shown in the figure, there is a rapid increase in the temperature of the heating unit and the working medium at the outlet of the evaporator when the system starts up. This is attributed to the low specific heat capacity of the liquid working medium. When the single-phase flow changes to a two-phase flow, obvious temperature fluctuations appear; under some conditions, the evaporator temperature overshoot phenomenon occurs, which is a critical metastable state. In contrast, when the working medium starts to gasify, the rate of temperature rise slows due to the high required gasification latent heat. With further augmentation of the input heat power density, the working medium temperature at the outlet of the evaporator correspondingly increases until it approaches the saturation steam temperature. It is noteworthy that the difference between the inlet and outlet temperatures of the 
evaporator is approximately $30^{\circ} \mathrm{C}$, which indicates the high efficiency of two-phase flow heat transfer. However, when the input power density increases from $150 \mathrm{~W} / \mathrm{cm}^{2}$ to $187.5 \mathrm{~W} / \mathrm{cm}^{2}$, the evaporator undergoes a great step change in temperature. The explanation is that the critical heat flux density of this circumstance is between $150 \mathrm{~W} / \mathrm{cm}^{2}$ and $187.5 \mathrm{~W} / \mathrm{cm}^{2}$; once the input power density exceeds this critical value, the evaporator shifts from nuclear boiling to film boiling, and the wall surface is covered by a layer of hot gas film, leading to the deterioration of the heat transfer between the working medium and evaporator.

The heat load also has a significant influence on the pressure response at the outlet of the evaporator. The pressure evolution at the evaporator outlet can be divided into four stages: steady state, drastic oscillation, damped oscillation, and slight oscillation. During the steady-state stage, the pressure is nearly unchanged due to the steady flow of the liquid working medium, and the heat transfer mechanism is mainly forced convection heat transfer. However, the outlet pressure evolution of the evaporator in the two-phase flow stage is quite different from that in the steadystate stage. Figure $\mathbf{3 B}$ shows the pressure fluctuations at the evaporator outlet during different stages of two-phase flow. During the early stage of the two-phase process, the pressure oscillations are drastic due to unsteady bubble generation and motion. The continuous small bubbles combine in clusters to form larger bubbles and then burst or stretch because of bubble motion, which all contribute to the large-amplitude pulsation at the outlet pressure of the evaporator (see Figure 3Bi). The damped oscillation of the evaporator outlet pressure is observed in Figure 3Bii. The explanation is that the increase in bubbles leads to the formation of a large bubble column in the channel center, and the liquid working medium is pushed against the channel wall, where it forms a local liquid film, thereby inhibiting the further formation of bubbles and leading to attenuation of the pressure oscillation at the evaporator outlet (i.e., the pressure oscillation amplitude changes from $17 \mathrm{kPa}$ to $2 \mathrm{kPa}$ ). Figure 3Biii shows that the pressure oscillation is finally slight (i.e., the pressure oscillation amplitude is $1.5 \mathrm{kPa}$ ) as the system enters a stable annular flow heat transfer mode, where a stable liquid film is attached to the pipe wall with the steam core zone in the middle. The heat transfer at this mode is mainly achieved by forced convective evaporation of this liquid film, and the heat transfer efficiency of this heat exchange mode is very high. Moreover, as the heat flux density increases, the liquid film becomes thinner, and the heat transfer efficiency further improves. However, it is noteworthy that after the system is started, the increase in input power density correspondingly increases the system pressure but is independent of the pressure oscillations.

\section{REFERENCES}

Chen, Y., Liu, X., and Shi, M. (2013). Hydrodynamics of Double Emulsion Droplet in Shear Flow. Appl. Phys. Lett. 102 (5), 051609. doi:10.1063/1. 4789865

\section{CONCLUSION}

In the present work, an MPCL system was constructed to perform an experimental investigation on the dynamic heat transfer characteristics of the MPCL system, including the start-up characteristics and variable-condition characteristics. The dynamic thermal response to the heat load and the pressure oscillation were examined and analyzed. The main conclusions of this study are summarized as follows:

(1) During the start-up period, pressure oscillation and temperature overshoot phenomena occur at the evaporator outlet when the system loop shifts from a single phase to two phases. An increase in the input power density leads to a decrease in the temperature overshoot and duration time of adjustment while resulting in an augmentation in the amplitude of pressure oscillations.

(2) During the regular operation period, with the augmentation of the input power density, the outlet temperature of the evaporator gradually increases until it approaches the saturation temperature and then remains constant. The pressure evolution at the evaporator outlet can be divided into four stages: steady state, drastic oscillation, damped oscillation, and slight oscillation. However, once the input power density exceeds the critical heat flux density under these circumstances, the evaporator temperature no longer increases slowly but undergoes a great step-like increase.

\section{DATA AVAILABILITY STATEMENT}

The original contributions presented in the study are included in the article/Supplementary Material, further inquiries can be directed to the corresponding author.

\section{AUTHOR CONTRIBUTIONS}

LL, JT, and WG contributed to conception and design of the study. JT performed the experiments and wrote the first draft of the manuscript. All authors contributed to manuscript revision, read, and approved the submitted version.

\section{ACKNOWLEDGMENTS}

This work was supported by the Postgraduate Research \& Practice Innovation Program of Jiangsu Province (KYCX19_0076).

Chen, Y., Zhang, C., Shi, M., and Yang, Y. (2009). Thermal and Hydrodynamic Characteristics of Constructal Tree-Shaped Minichannel Heat Sink. Aiche J. 56 (8), 2018-2029. doi:10.1002/aic.12135

Deng, Z., Zhang, J., Lei, Y., Tao, J., and Zhang, C. (2021). Startup Regimes of Minichannel Evaporator in a Mechanically Pumped Fluid Loop. Int. J. Heat Mass Transfer 176, 121424. doi:10.1016/j.ijheatmasstransfer.2021.121424 
Jie, L., Mou, L., shuang-xi, Z., Kai-hua, G., Zhen-hui, H., and Ting-xuen, L. (2008). Experimental Investigation on Start-Up of Mechanically Pumped Cooling Loop. Energ. Convers. Manag. 49 (10), 2595-2601. doi:10.1016/j.enconman. 2008.05.007

Kloczko, S., and Faghri, A. (2020). Experimental Investigation on Loop Thermosyphon thermal Performance with Flow Visualization. Int. J. Heat Mass Transfer 150, 119312. doi:10.1016/j.ijheatmasstransfer. 2020.119312

Lee, H., Park, I., Mudawar, I., and Hasan, M. M. (2014). Micro-channel Evaporator for Space Applications - 1. Experimental Pressure Drop and Heat Transfer Results for Different Orientations in Earth Gravity. Int. J. Heat Mass Transfer 77, 1213-1230. doi:10.1016/j.ijheatmasstransfer.2014.06.012

Liu, X., Chen, Y., and Shi, M. (2013). Dynamic Performance Analysis on Start-Up of Closed-Loop Pulsating Heat Pipes (CLPHPs). Int. J. Therm. Sci. 65, 224-233. doi:10.1016/j.ijthermalsci.2012.10.012

Liu, X., Zhang, C., Yu, W., Deng, Z., and Chen, Y. (2016). Bubble Breakup in a Microfluidic T-junction. Sci. Bull. 61 (10), 811-824. doi:10.1007/s11434-0161067-1

Ma, Y., Ma, G., Zhang, S., and Zhou, F. (2016). Cooling Performance of a PumpDriven Two Phase Cooling System for Free Cooling in Data Centers. Appl. Therm. Eng. 95, 143-149. doi:10.1016/j.applthermaleng.2015.11.002

Maydanik, Y. F. (2005). Loop Heat Pipes. Appl. Therm. Eng. 25 (5), 635-657. doi:10.1016/j.applthermaleng.2004.07.010

Meng, Q., Zhao, Z., Zhang, T., van Es, J., Pauw, A., Zhang, H., et al. (2020). Experimental Study on the Transient Behaviors of Mechanically Pumped Twophase Loop with a Novel Accumulator for thermal Control of Space Camera Payload. Appl. Therm. Eng. 179, 115714. doi:10.1016/j.applthermaleng.2020. 115714
Shi, X., Yin, B., Chen, G., Zhang, X., and Mei, X. (2021). Numerical Study on Twophase Flow and Heat Transfer Characteristics of Loop Rotating Heat Pipe for Cooling Motorized Spindle. Appl. Therm. Eng. 192, 116927. doi:10.1016/j. applthermaleng.2021.116927

Thome, J. R. (2006). State-of-the-Art Overview of Boiling and Two-phase Flows in Microchannels. Heat Transfer Eng. 27 (9), 4-19. doi:10.1080/ 01457630600845481

Vasiliev, L. L. (2005). Heat Pipes in Modern Heat Exchangers. Appl. Therm. Eng. 25 (1), 1-19. doi:10.1016/j.applthermaleng.2003.12.004

Wang, C., Yao, F., Shi, J., Wu, L., and Zhang, M. (2018). Visualization Study on Thermo-Hydrodynamic Behaviors of a Flat Two-phase Thermosyphon. Energies 11, 2295. doi:10.3390/en11092295

Zhang, C., Li, G., Sun, L., and Chen, Y. (2021). Experimental Study on Active Disturbance Rejection Temperature Control of a Mechanically Pumped Twophase Loop. Int. J. Refrigeration. doi:10.1016/j.ijrefrig.2021.04.038

Zhou, B., Cai, P., and Chen, Y. (2019). Interfacial Mass Transfer of Water for Fluorobenzene/aqueous Solution System in Double Emulsion. Int. J. Heat Mass Transfer 145, 118690. doi:10.1016/j.ijheatmasstransfer.2019.118690

Zhu, L., and Yu, J. (2019). Optimization of a Separator Assisted Two-phase Thermosyphon Loop by Using Entropy Generation Analysis. Int. J. Refrigeration 98, 15-24. doi:10.1016/j.ijrefrig.2018.09.029

Copyright (c) $2021 \mathrm{Li}$, Tao and Gao. This is an open-access article distributed under the terms of the Creative Commons Attribution License (CC BY). The use, distribution or reproduction in other forums is permitted, provided the original author(s) and the copyright owner(s) are credited and that the original publication in this journal is cited, in accordance with accepted academic practice. No use, distribution or reproduction is permitted which does not comply with these terms. 